\title{
El Instituto Politécnico Nacional como educación popular: la fuerza de una idea, $1942^{1}$
}

The National Polytechnic Institute as Popular Education: The Strength of an Idea, 1942

\author{
Andrés Ortiz Morales \\ Instituto Politécnico Nacional \\ aortizmo@ipn.mx \\ ortizandy2001@yahoo.com.mx
}

\begin{abstract}
El objetivo de este trabajo es identificar las causas que llevaron a un sector de la sociedad a enfrentar a las autoridades educativas, en 1942, debido a la pretensión de desintegrar el Instituto Politécnico Nacional (IPN) cuando éste apenas rebasaba un lustro de existencia. Se revisó el contexto de la creación del instituto y el proyecto político, económico y social de izquierda radical que lo arropó el cual entró en conflicto con el programa de unidad nacional que el presidente Manuel Ávila Camacho instrumentó en el marco de la segunda Guerra Mundial. Se encontró que durante el cardenismo se construyó el discurso que mostraba al IPN como conquista social de la Revolución mexicana, lo que de paso proporcionaba un elemento de legitimidad para la nueva élite "revolucionaria". Para los sectores movilizados (alumnos, profesores, sociedad capitalina, sindicatos nacionales), el IPN representaba una de las pocas expresiones tangibles de la anhelada justicia social que prometió la Revolución, era la posibilidad real de acceder a los beneficios del progreso que, a cuentagotas, llegaba a los sectores marginados. Esta idea resultó bastante significativa, más cuando consideramos que ha perdurado luego de 80 años de la creación de esta entidad educativa de carácter tecnológico-científico.
\end{abstract}

Resumen

Palabras clave: Instituto Politécnico Nacional, Revolución mexicana, cardenismo, educación técnica, huelga estudiantil.

${ }^{1}$ Este artículo se relaciona con el proyecto "Construcción del modelo educativo y científico-tecnológico: IPN (1930-1940). Influencia de los sistemas técnico-tecnológicos extranjeros" desarrollado en el Programa Especial de Consolidación y Formación de Grupos de Investigación, registro SIP 20161969, de la Secretaría de Investigación y Posgrado del Instituto Politécnico Nacional. 


\section{Abstract}

The aim of this article is to identify the causes that led a sector of society to confront educational authorities in its attempt to disintegrate the National Polytechnic Institute (IPN) in 1942, when it had barely a decade of existence. In order to do so, I revise the context of the creation of the Institute, the radical political, economic and social project into which it was framed, which then came into conflict with the program of national unity which was implemented by President Manuel Ávila Camacho against the backdrop of the Second World War. I show that during the "Cardenismo", an official discourse was articulated that featured the IPN as a social conquest of the Mexican Revolution, which by the way, provided an element of legitimacy for the new "revolutionary" elite.

For the sectors mobilized in 1942 (students, teachers, inhabitants of Mexico City, national trade unions), the IPN represented one of the few tangible expressions of the social justice promised by the Revolution. It represented a real possibility for the marginalized sectors of society to access the benefits of progress. This idea proved quite significant, especially when we consider that 80 years after the creation of this educational institution of technological-scientific, it still prevails.

Keywords: National Polytechnic Institute, Mexican Revolution, Cardenismo, technical education orientation, technical education, student strike.

\section{Introducción}

A inicios del periodo presidencial de Lázaro Cárdenas (1934-1940) las discrepancias entre éste y Plutarco Elías Calles pronto se manifestaron. El también llamado "jefe máximo de la Revolución" consideraba que el país necesitaba fortalecer la iniciativa privada, brindar garantías a la propiedad privada y detener el reparto de tierra comunal; por ello los gobiernos que estuvieron bajo su influencia dieron estímulos a la producción individual y buscaron un clima de estabilidad interior para ofrecer seguridad a las inversiones nacionales y extranjeras, aunque mediante la represión de los movimientos agrario y obrero. Para los gobiernos de los presidentes sonorenses (1920-1934) las reformas sociales plasmadas en el texto constitucional de 1917 fueron letra muerta.

Si bien la política progresista tendría continuidad en el periodo del presidente Cárdenas, éste visualizó un camino diferente para lograr un Estado fuerte. Consideraba que el campesinado y el sector obrero debían constituir las bases sociales del poder, por tanto, apoyó el agrarismo y los derechos laborales y fue firme impulsor del nacionalismo. La ruta que trazó era reflejo de su experiencia en la lucha armada y en la política, pero también estuvo determinada por la situación social del país. Aunque dividido y debilitado, hacia 1934 el movimiento 
obrero se había hecho más radical, un problema heredado por Cárdenas quien tuvo la habilidad de encauzarlo y convertirlo en fuerza política al crear, en 1936, la Confederación de Trabajadores de México (CTM) empleando un discurso en el que proponía un equilibrio justo entre capital y trabajo.

Mediante el reparto ejidal, para cumplir con la reforma agraria, el gobierno ganó la simpatía y la lealtad campesina; logró incorporar en el proyecto de nación a la sociedad rural, mayoritaria en México, con giras por el interior del país y tomando nota de las necesidades de cada región. Ocurrió que, por primera vez, el gobierno adoptaba los preceptos sociales adscritos en la Constitución, mismos que fueron incluidos en el Plan Sexenal, documento elaborado en 1933 por la Comisión de Colaboración Técnica del Partido Nacional Revolucionario (PNR) con el fin de establecer directrices al gobierno, de 1934 a 1940. De esta manera, las circunstancias políticas fueron favorables para que la administración de Cárdenas brindara un fuerte apoyo a los sectores populares, al menos hasta 1938 año en el que se frenó dicha tendencia.

Durante la década de 1920 y los primeros años de la siguiente, las autoridades educativas se referían constantemente a la enseñanza técnica como una reivindicación popular lograda por la Revolución. Sin embargo, en el contexto del estancamiento de la puesta en marcha de las reformas sociales, de la crisis económica mundial del sistema capitalista a partir de 1929, que repercutía en la economía mexicana, y del arribo al país de inquietudes socialistas - para algunos políticos no pasaba desapercibida la fortaleza frente a la crisis por parte de la Unión Soviética-, al interior del PNR se conformó una propuesta radical en contra de toda educación fundada en creencias religiosas y en favor del establecimiento de una educación socialista. Esta tendencia se expresó en la propuesta de reforma al artículo $3^{0}$ constitucional, encabezada por Alberto Bremauntz, la cual fue aprobada por ambas cámaras en octubre de 1934 y entró en vigor en diciembre del mismo año. El párrafo inicial quedó de la siguiente manera: "La educación que imparta el Estado será socialista y, además de excluir toda doctrina religiosa, combatirá el fanatismo y los prejuicios, para lo cual la escuela organizará sus enseñanzas y actividades en forma que permita crear en la juventud un concepto racional y exacto del universo y de la vida social". 2 Los reformadores intentaron una profunda transformación del sistema educativo nacional, pues sujetaron la educación particular a los programas oficiales en el entendido de que a través de la educación sería posible estimular un proceso de cambio social que repercutiera en lo económico y lo político; su planteamiento manifestaba la intención de retomar el rumbo social de la Revolución.

Los nuevos funcionarios de la Secretaría de Educación Pública (SEP) señalarían que aún prevalecía el carácter elitista de la educación, incluso en las escuelas técnicas. El secretario

2 "Decreto que reforma el Artículo $3^{\circ}$ y la fracción xxv del 73 constitucionales", Diario Oficial de la Federación (DOF), 13 de diciembre de 1934, p. 1. 
de Educación, Narciso Bassols (octubre de 1931 a mayo de 1934), encabezó un equipo de trabajo que luego de realizar un diagnóstico encontró que "el sistema de reclutamiento de estudiantes para las escuelas técnicas es el sistema democrático de enseñanza gratuita [...] este sistema permite que se opere libremente una selección económica desfavorable [...] a todos los trabajadores y a los hijos de todos los trabajadores de salario bajo y favorable a la pequeña burguesía y a la clase media urbana" (SEP, 1932: 369). Se señalaba que no había manera de que los jóvenes trabajadores pudieran dedicar 36 o 40 horas semanales al estudio, ni que los padres financiaran a sus hijos desde los 14 hasta los 22 años, que es el tiempo que les tomaría graduarse como profesionistas.

\section{Creación del Instituto Politécnico Nacional}

En opinión de los funcionarios de postura radical, la escuela técnica debía estar vinculada a los grandes grupos de la población: campesinos, obreros y otros sectores marginados para cumplir así con la meta social de la Revolución. Desde la Secretaría se elaboró la propuesta para construir un sistema articulado de educación técnica llamado Escuela Politécnica Mexicana, concebida a partir del conjunto de escuelas ya existentes. El plan fue enviado para su revisión a la Comisión Técnica Consultiva de la SEP, el 22 de enero de 1932. En el Reglamento de alumnos para las escuelas preparatorias técnicas y de estudios técnicos superiores que integran la Escuela Politécnica Nacional (1933) se señalaba que la institución marcaría un avance en el sentido de ofrecer al pueblo altas posibilidades educativas, pues comprendía niveles de formación de obrero, técnico y profesionista.

La reorganización atravesó por diferentes etapas en poco más de cuatro años y culminó con la creación del Instituto Politécnico Nacional (IPN) el cual inició labores en 1936. El $1^{\circ}$ de enero de 1937, Gonzalo Vázquez Vela, titular en turno de la SEP, nombró "jefe de instituto" a Roberto Medellín Ostos y en una ceremonia realizada en el Palacio de Bellas Artes de la Ciudad de México el IPN inauguró oficialmente sus cursos el 20 de febrero de 1937. ${ }^{3}$

El Politécnico era todo un subsistema de educación que contaba con escuelas prevocacionales (posprimarias), escuelas vocacionales (preparatorias técnicas) y escuelas superiores algunas de las cuales tenían su equivalente en la Universidad Autónoma de México. ${ }^{4}$ Así ocurría con las escuelas de Medicina, Ingeniería y Arquitectura. Hay que recor-

\footnotetext{
3 "Los cursos en las Escuelas Politécnicas se inauguraron ayer en todos los planteles que dependen del Instituto Politécnico Nacional", El Nacional, 21 de febrero de 1937, p. 2.

${ }^{4}$ La Universidad se distanció del Estado que emanó de la Revolución; en 1933 se le retiró el carácter de nacional, una estrategia del gobierno para aislarla debido a su oposición a la política educativa socialista. Recuperó su denominación de Universidad Nacional Autónoma de México (UNAM), en 1945.
} 
dar que la Universidad "llegó a ser un actor esencial en el marco de la resistencia a las políticas educativas socialistas del Estado" (Ordorika, 2006: 72), pues los universitarios asumieron como una responsabilidad el adquirir y difundir el conocimiento en un sentido imparcial, con libertad académica de investigación, exposición y enseñanza, así que se enfrentaron a la postura de los funcionarios cardenistas que les exigían compromiso social y adoptar una filosofía en favor de los sectores populares y del progreso material del país.

En el nivel superior, el IPN integró siete escuelas durante su primer año: del área de ciencias exactas y fisicoquímicas aplicadas, las Escuelas Federales de Industrias Textiles 1 y 2 , antecedente de la Escuela Superior de Ingeniería Textil (ESIT); la Escuela Superior de Construcción, hoy Escuela Superior de Ingeniería y Arquitectura (ESIA); y la Escuela Superior de Ingeniería Mecánica y Eléctrica (ESIME). Del área de ciencias económicas y sociales incorporó a la Escuela Comercial para Señoritas Miguel Lerdo de Tejada y la Escuela Superior de Ciencias Económicas, Administrativas y Sociales, hoy Escuela Superior de Comercio y Administración (ESCA). Del área de ciencias biológicas aplicadas el IPN incorporó la Escuela de Medicina Homeopática, creada en 1895, hoy Escuela Nacional de Medicina y Homeopatía (ENMH). Al correr el año de 1936 se sumó la Escuela Nacional de Bacteriología, Parasitología y Fermentaciones, hoy Escuela Nacional de Ciencias Biológicas (ENCB).

\section{EI IPN y la educación popular}

El discurso del gobierno cardenista exaltaba la idea de la educación pública como un medio para arribar al progreso social, postulado atrayente para los sectores marginados. Se insistía en que la educación media y superior debían ser más prácticas y menos teóricas, más vinculadas al trabajo productivo y a la atención de los problemas nacionales que a la reflexión intelectual. Respecto a su carácter popular destacaba:

El Instituto Politécnico Nacional propende hacia la formación de un amplio sistema de enseñanzas profesionales, sin que lo guíe el afán de repetir o contradecir lo existente, sino simplemente acercar la enseñanza superior a las grandes masas sociales de México y satisfacer las ingentes necesidades colectivas, con la formación de profesionistas capaces e identificados con las aspiraciones que arrancan de nuestra nacionalidad (SEP, 1941: 451)

En el IPN las carreras de médico, ingeniero, arquitecto, contador, enfermera, biólogo, químico y técnico en diversas áreas estaban al alcance de la gente humilde cuyos hijos antes no tenían acceso, ni a estudiarlas ni a los beneficios de su ejercicio. En la Memoria SEP de 1934 se anota: "No podrá ser efectiva la eficacia de la enseñanza técnica, dentro de la actual organización del Estado, mientras no se hayan canalizado sus orientaciones para servir a los hombres que 
[...] pertenezcan a la clase productora, a la que debe estar dirigida íntegramente la enseñanza de este tipo" (SEP, 1934: 184). Al incluir estas carreras en el Politécnico, el Estado simbólicamente daba por abolido el privilegio de la educación superior exclusiva para las élites. De acuerdo con los datos registrados en el Anuario de 1939, la población escolar del IPN se componía 29\% por hijos de empleados oficiales o particulares y $25 \%$ por hijos de obreros, campesinos o artesanos, mientras que 10\% no tenía padre o tutor que sostuviese sus estudios (SEP, 1939: 12). Al respecto, el historiador Humberto Monteón González (1986: 9) señala que de 1936 a 1939 un promedio anual de 71\% de los alumnos del IPN pertenecía a los sectores desfavorecidos de la sociedad, ya fueran del campo o de la ciudad, y que en algunos casos los jóvenes tenían que estudiar y trabajar. Frente a este número de estudiantes se hallaba un 26\% que pertenecía al sector de clase media.

En este sentido debemos destacar que Luis Enrique Erro, jefe del Departamento de Enseñanza Técnica, Industrial y Comercial (DETIC), consideraba que la educación superior en México tenía dos orientaciones sociales: la "universitaria, y que sería reservada para aquellos profesionistas con un cierto tinte burgués, que además de poder pagar más dinero por la enseñanza recibida, llevarán como finalidad el conocimiento de las humanidades" (SEP, 1932: 429); y siguiendo su idea, frente a esta modalidad la revolución había creado la opción del IPN donde "la enseñanza profesional tiene que ser idéntica para toda clase de individuos; no determinan sus modalidades las diferencias sociales; la meta, el conjunto de conocimientos, para resolver problemas perfectamente determinados" (SEP, 1932: 429). Esta idea planteaba la división del sistema educativo al acotar los estudios y las escuelas a las que cada individuo debería asistir, dependiendo de su situación económica: burgués-Universidad, proletarioPolitécnico.

\section{EI IPN para atender las necesidades del mundo industrial}

Por otra parte, al igual que otros países latinoamericanos, México fue afectado por la crisis de 1929. Al perder los mercados para las materias primas entre los países industriales (Urquidi, 2005: 64), así como las fuentes de abasto de manufacturas, se hizo necesario que el gobierno adoptara medidas de tipo comercial, financiero y fiscal. Así inició el proceso tendente a crear un mercado interno para los productos del país, protegido con altos aranceles y restricciones a las importaciones (Urquidi, 2005: 82), y comenzó la aplicación de una política económica de orientación hacia adentro; se aumentó el gasto y se pusieron en marcha programas de obras públicas. La caída del mercado mundial tras la crisis, las tensas relaciones internacionales que precedieron a la segunda Guerra Mundial, la escasez de productos para la incipiente industria nacional -maquinaria, instrumentos, refacciones, productos intermedios tales como acero, sustancias químicas y combustibles - y de productos alimenticios y farmacéuticos 
fueron circunstancias que propiciaron una coyuntura histórica favorable para el inicio de la industrialización bajo la dirección centralizada del gobierno como principal inversionista, organizador y promotor.

De esta manera, en el periodo cardenista el Estado hizo suyas la generación de electricidad y las comunicaciones eléctricas, áreas que habían estado en manos de la iniciativa privada, generalmente de empresarios extranjeros. La nacionalización de los ferrocarriles (1937) y el petróleo (1938) resultó vital para impulsar el sector industrial, que en 1929 había aportado 18.6\% del ingreso nacional — superado por la agricultura y la minería — y que para 1939 ocupaba el primer puesto con 23.1\% del total (Ruiz, 2004: 155).

La incipiente industrialización basada en nuevas tecnologías e instrumentos tecnológicos, la creación de empresas paraestatales, las nacionalizaciones, el reparto agrario, la creación de infraestructura, todo ello en conjunto planteó al gobierno cardenista la necesidad de instrumentar aplicaciones técnicas de los conocimientos científicos y tecnológicos; y las autoridades argumentaron que la modernización del país pondría fin a la explotación, por parte de las potencias extranjeras, tanto de los recursos naturales como de la mano de obra. Ignacio García Téllez, secretario de Educación entre 1934 y 1935 acotó que la creación del IPN debería incidir en "[...] la sustitución de elementos extranjeros por nacionales y la creación de una industria nacional” (Rodríguez y Krongold, 1988: 51). En este contexto la educación técnica fue considerada moderna por excelencia porque se correspondía con la industrialización capitalista, se sustentaba en el desarrollo científico y se consideraba que sería la base sobre la cual el país desarrollaría su economía. En el fondo estaba la voluntad por liberar las fuerzas productivas de la nación bajo la idea de "regenerar" a las mayorías incorporándolas a la modernidad, es decir, en el sistema de valores favorables a la economía capitalista.

\section{El giro a la derecha y las presiones al Politécnico}

El $1^{\circ}$ de diciembre de 1940 Manuel Ávila Camacho relevó a Lázaro Cárdenas en la presidencia para el periodo 1940-1946. Desde finales de 1938 el gobierno cardenista había abandonado el radicalismo de izquierda y reorientaba sus acciones hacia la industrialización del país, correspondiendo al Estado la creación de obras de infraestructura y a los particulares la generación de nuevas fábricas (González, 2005: 268). Se eliminaron impuestos a ciertas industrias, se frenaron los movimientos huelguísticos y se fomentó la exportación de productos hechos en México. Ávila Camacho inició un gobierno moderado que se enfocaría en promover la unidad nacional dadas las tensiones al interior de la sociedad por el rechazo de los pequeños propietarios y de la burguesía, así como de otros sectores conservadores 
influenciados por la Iglesia católica, a las reformas radicales, y al exterior debido a la presión estadounidense ante la expropiación petrolera.

A diferencia del gobierno de Cárdenas la administración de Ávila Camacho reorientó la base social del poder del Estado hacia los sectores medios urbanos, a los empresarios y a la creciente burocracia. La segunda Guerra Mundial, como amenaza externa, sirvió para justificar el abandono del reformismo cardenista y la implantación de una política conciliadora y de unidad nacional; la administración hacía hincapié en los valores nacionales y enfáticamente rechazaba el comunismo (Knight, 1988: 10). La intención del gobierno era recuperar la confianza de los inversionistas, actores principales en el proyecto desarrollista industrial, lo que se concretó con el incremento de las inversiones estadounidenses, dirigidas principalmente a la industria manufacturera. El gobierno ya no buscaba fortalecer una economía popular, por lo contrario, se implementó una férrea política de contención salarial para fortalecer al capital privado, tarea en la que colaboró de modo eficaz la CTM. En el campo, la CNC se vio debilitada por el fomento de la propiedad privada dedicada a la agricultura comercial de exportación mientras que, por otra parte, se detuvo el reparto agrario y se favoreció la parcelación de los ejidos, todo bajo la postura gubernamental de neutralidad. De este modo quedó disminuida la política social en favor de la modernización.

En materia educativa Luis Sánchez Pontón, político radical que impulsó la educación socialista, fue sustituido al frente de la SEP por el abogado Octavio Véjar Vázquez en septiembre de 1941. Este último denominó a su política educativa "escuela del amor" y rápidamente tomó postura en contra de la educación popular-socialista. En palabras de Véjar: "Contra la furia desgreñada de los que han querido imponer caprichos, violentar conciencias, arrancarnos de la raíz vital de la tradición, y desgarrar el alma auténtica de México [...] la escuela debe reconocer como la más importante de sus funciones, la de ser elemento de unificación. Nada que divida puede ocupar sitio en sus aulas" (Junco, 1942: 5). A partir del $1^{\circ}$ de enero de 1942 Véjar reorganizó la Secretaría en ocho direcciones generales y tres oficinas, entre las cuales se encontraba la Dirección General de Enseñanza Superior e Investigación Científica. Al interior de ésta se creó un nuevo Departamento de Enseñanza Industrial y Comercial el cual debía ser administrado por el IPN. Sin embargo, lo que ocurrió fue que la nueva administración manifestó su intención de desaparecerlo.

En el aspecto económico se aplicó una abrupta reducción en los recursos asignados al instituto, la cual tocó fondo en 1942 ocasionando serios problemas operativos. De un presupuesto en 1940 de 8375000 pesos, hacia 1941 esta cifra sería de 3229000 pesos y descendería finalmente a 2708000 pesos en 1942 (SEP, 1944: 7; SEP, 1946: 22, 289; Pérez, 1984: 162-163).

En el ámbito legal, el Congreso de la Unión aprobó en enero de 1942 la nueva Ley Orgánica de la Educación Pública que fue expedida para normar el artículo $3^{\circ}$ constitucional. Respecto 
a las diversas escuelas vocacionales, en el capítulo XII, artículo 84 se señalaba que "La educación vocacional de acuerdo con las específicas inclinaciones y aptitudes de los educandos, tiene por objeto la elevación de su cultura integral y su preparación especializada para estudios técnicos o profesionales". ${ }^{5}$ Respecto a las escuelas profesionales, en el capítulo XIII, artículo 89, se consignaba que "la educación superior [técnica] tiene por objeto la formación de técnicos y profesionistas, mediante el estudio intensivo de las ciencias y de su aplicación con fines de utilización práctica”. El artículo 98 organizó la educación técnica en departamentos conexos: secundaria, vocacional y profesional. El problema fue que en el texto se omitió deliberadamente el término "Instituto Politécnico Nacional". Además, la ley abría explícitamente la posibilidad de que los particulares intervinieran en la educación y a la vez estableció que el Estado no impondría una determinada ideología a los educandos. Así se ignoró la educación socialista (la que finalmente sería desechada con la reforma al artículo tercero, en 1945).

A Wilfrido Massieu —nombrado director del IPN por Lázaro Cárdenas en mayo de 1939—, se le comunicó en enero de 1942 su designación como director de la vocacional 1, "por haber cesado en el puesto de director del Instituto Politécnico Nacional por desaparición del mismo".

Para inclinar a la opinión pública en contra del Politécnico, el secretario de Salubridad y Asistencia Pública, Gustavo Baz, continuó una campaña, iniciada en 1938 durante el $2^{\circ}$ Congreso de Higiene Rural, celebrado en San Luis Potosí, en contra de Miguel Othón de Mendizabal y Leopoldo Ancona Hernández, profesores de la Escuela Nacional de Ciencias Biológicas (ENCB), quienes presentaron el proyecto para formar al médico rural (Archivo Histórico Escuela Nacional de Ciencias Biológicas, 1938). Baz pretendía terminar con la carrera de Medicina Rural —impartida en la ENCB del IPN — a la que calificaba de fábrica de médicos al vapor. En un telegrama dirigido a la presidencia de la República, la sociedad de alumnos alertaba: "tales procedimientos del Dr. Gustavo Baz han originado agitación estudiantil [lo que] amenaza convertirse en movimiento general en su contra"? El mismo director del IPN, Wilfrido Massieu, tuvo que advertirle al presidente Ruíz Cortines sobre el incremento de la molestia de los alumnos politécnicos frente a estas acciones. En octubre de 1941 se formó un Comité de Defensa del instituto, encabezado por José A. Díaz de Sandi, en respuesta al cese de los profesores fundadores de la ENCB, Gerardo Varela y Alfonso Dampf, hecho que

\footnotetext{
5 "Ley Orgánica de Educación Pública, reglamentaria de los artículos 3; fracción I; 73, fracciones x y xxV; y 123, fracción XII, de la Constitución Política de los Estados Unidos Mexicanos", Diario Oficial de la Federación, 23 de enero de 1942, p. 26.

${ }^{6}$ AHC-IPN (1942), "Acuerdo de Roberto T. Bonilla, subsecretario de Educación Pública, al director general de administración", 14 de enero de 1942, Archivo Histórico Central del Instituto Politécnico Nacional, Ciudad de México, Fondo Colección Especial, Sección Wilfrido Massieu.

${ }^{7}$ AHC-IPN (1941-1942), "Compendio de noticias con referencia al movimiento estudiantil de 1942", t. I, Archivo Histórico Central del Instituto Politécnico Nacional, Ciudad de México, Hemeroteca, p. 16.
} 
se tomó como una agresión al instituto. En documento firmado por profesores como Miguel Othón de Mendizábal, Rodolfo Hernández Corzo, así como la Asociación de Químicos Bacteriólogos, se solicitó la mediación del presidente ante la Secretaría de Educación (Rodríguez y Krongold, 1988: 112).

El 21 de enero de 1942 se sumaría a esas acciones la publicación del Acuerdo 530, signado por el secretario de Educación, donde se ordenaba que el Departamento de Antropología de la ENCB quedara adscrito al Instituto Nacional de Antropología e Historia (INAH) (López, 2011: 35). El IPN cedió así las carreras de Antropólogo Físico y Antropólogo Social, que ya tenía estructuradas. Para exasperar aún más a la comunidad politécnica se corrió el rumor de que los títulos expedidos por los planteles del Politécnico deberían ser avalados en el futuro por la Universidad Nacional Autónoma de México (UNAM), lo que se fundaba en declaraciones como la siguiente, del rector Mario de la Cueva: "En la inauguración de cursos de 1941, al dirigirme por primera vez a los estudiantes, en mi carácter de rector, les dije que un día las universidades de la República y el Instituto Politécnico Nacional habrían de reconocer la majestad de nuestra institución. Esperamos que definitivamente sostengan nuestros postulados y que sigan nuestra senda de superación y honestidad". ${ }^{8}$

En otras instancias del sistema educativo también ocurría la persecución de Véjar Vázquez en contra de los profesores y directivos que apoyaban el proyecto educativo cardenista, ahora denominados "agitadores comunistoides", muchos de los cuales fueron cesados de sus puestos a lo largo y ancho del país. ${ }^{9}$ Conviene subrayar que las Escuelas Regionales Campesinas también sufrieron el embate avilacamachista a la educación popular, ya que fueron fragmentadas en Escuelas Prácticas de Agricultura, para la formación de técnicos agrícolas, y en Escuelas Normales Rurales, para los maestros; se eliminaron los internados mixtos y se redujo la participación de la escuela normal en la comunidad, al imponérseles un plan de estudios similar al de las normales urbanas (Civera, 2013: 317). La nueva administración consideró necesario racionalizar la educación popular, por lo que se practicó una selección rigurosa de ingreso a las instituciones educativas: se hizo a un lado a los maestros con antecedentes de participación política, principalmente en las normales, en las escuelas regionales campesinas y en las escuelas técnicas, consideradas focos de confrontación con el nuevo modelo de la educación (Gómez, 2003: 193).

En este orden de ideas tenemos que se pensó en desmantelar al IPN sin cerrar los planteles que estaban en funciones. La lógica era que la escuela ya no asumiera una postura de combate social, en contra de lo que el ala radical del gobierno cardenista había alentado antes. Al estudiar esta situación, Guevara Niebla (1980: 57) apunta que la nueva adminis-

8 "Cuando luche la juventud, que sea por su decoro", El Universal, 8 de marzo de 1942, p. 1.

9 "Los causantes de la agitación estudiantil", editorial; "Muchos alumnos de las escuelas técnicas están contra la huelga", El Universal, 6 de marzo de 1942, pp. 1 y 4. 
tración abandonó desde comienzos del sexenio la orientación socialista de la educación porque pensó que sus principios fomentaban antagonismos, considerados por las autoridades como inútiles, desgastantes y peligrosos. Si bien las escuelas agrupadas en el IPN resultaban vitales para atender la necesidad de obreros calificados, técnicos e ingenieros que demandaba la incipiente industrialización del país, así como la operación de la industria petrolera y la generación de electricidad, el Politécnico también representó un problema político debido al sesgo popular que le imprimió el régimen cardenista en su discurso.

En el esquema industrializador de la economía se convirtieron en objetivos primordiales el crecimiento de la planta productiva moderna y el aumento de la productividad, por lo que la atención se volcó hacia la universidad, al considerar que respondía mejor a los proyectos de expansión y mejoramiento de la clase media urbana y a la demanda de profesionistas. De esta manera fue que la institución recibió más recursos económicos y sus egresados tuvieron cada vez mayor presencia en la administración pública.

\section{La huelga estudiantil de marzo de 1942}

La respuesta del estudiantado del IPN fue enérgica. La Federación Nacional de Estudiantes Técnicos (FNET) conformó un Comité de Huelga encabezado por Francisco Moreno Salamanca e integrado por Gustavo Alvarado Pier, Jorge Ojeda, Alfonso Rus Quintal, Luis Camelo, Ernesto Moreno Bello, entre otros, quienes declararon la huelga el 4 de marzo de 1942 al mediodía. ${ }^{10}$ La FNET demandaba legalizar la existencia del Politécnico asignándole una ley orgánica, reintegrar las escuelas prevocacionales al IPN, dotar de laboratorios, bibliotecas, y talleres a las escuelas; continuar las construcciones del internado, del hospital de Medicina Rural, del auditorio del IPN y del edificio de la Escuela Superior de Ingeniería y Arquitectura (ESIA); reglamentar la carrera de Medicina Rural, reinstalar en la ESIA la carrera nocturna de Ingeniero Topógrafo y aplicar el presupuesto para satisfacer las necesidades de los alumnos del IPN."

El 6 de marzo se organizó una manifestación cuyo propósito fue solicitar al presidente de la República que detuviera el acoso al IPN y que su gobierno diera marcha atrás a las reformas que amenazaban su existencia, para lo cual los estudiantes se dirigieron a Palacio Nacional. Pero en la calle de Madero, frente al Zócalo de la capital, la policía y los bomberos recibieron órdenes de disolver la manifestación. Para no mojar la bandera de México que portaba la vanguardia estudiantil, se ordenó que unos policías montados se la arrebataran a sus portadores, con lo que se inició un enfrentamiento en el que hubo golpes, palos, pedradas,

\footnotetext{
${ }^{10} / \mathrm{dem}$.

${ }^{11}$ AHC-IPN (1941-1942), Compendio de noticias con referencia al movimiento estudiantil de 1942, t. I, Archivo Histórico Central del Instituto Politécnico Nacional, Ciudad de México, Hemeroteca, pp. 108-109.
} 


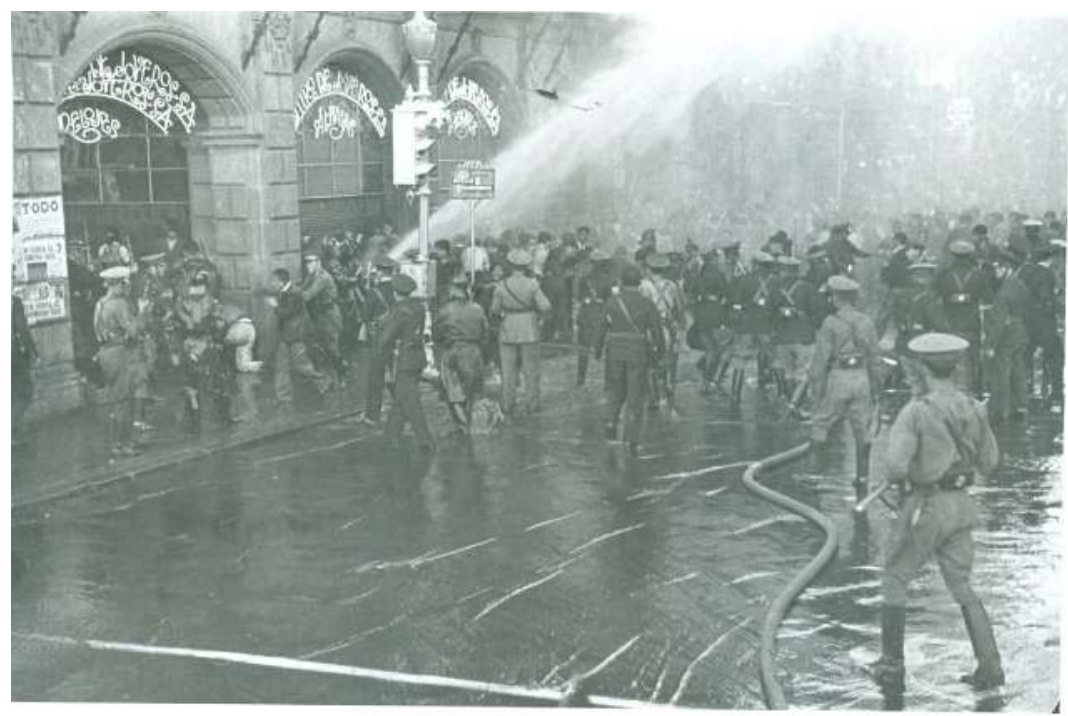

Fotografía 1. Los alumnos fueron dispersados por el cuerpo de bomberos y la policía. AHC-IPN, Fototeca, 6 de marzo de 1942

disparos y uso de armas blancas con un resultado de 18 heridos y dos víctimas mortales entre los manifestantes y las fuerzas del orden.12

Los representantes del IPN consiguieron una audiencia con el presidente en Palacio Nacional el 10 de marzo de 1942. Manuel Ávila Camacho les aseguró que "todas aquellas peticiones cuya resolución determine el mejoramiento de las escuelas que integran el instituto y los acuerdos que sean pedagógica y moralmente benéficos para los alumnos, serán resueltos dentro de un plano de absoluta justicia". ${ }^{13}$ Aunque los politécnicos se manifestaron satisfechos tras la audiencia y dos días después reanudaron las clases, la situación de incertidumbre continuó respecto a su posición dentro del sistema educativo. Por ello, el 30 de diciembre de 1942, en la Cámara de Diputados, el diputado Antonio Betancourt Pérez cuestionó al secretario de Educación Véjar Vázquez:

Se ha dicho que la Secretaría de Educación Pública ha realizado la desarticulación del Instituto Politécnico Nacional, convirtiéndolo, de un centro de investigación científico tecnológico y de alta formación profesional técnica, en varias escuelas de importancia inferior con tendencia a degenerar en escuela de oficios, típicas del artesano medieval

12 "Zafarrancho entre policías y estudiantes; el saldo fue de 2 muertos y 18 heridos", El Universal, 7 de marzo de 1942, p. 1

13 "El ejecutivo dará una solución justa a las peticiones escolares", El Nacional, miércoles 11 de marzo de 1942, p. 1. 


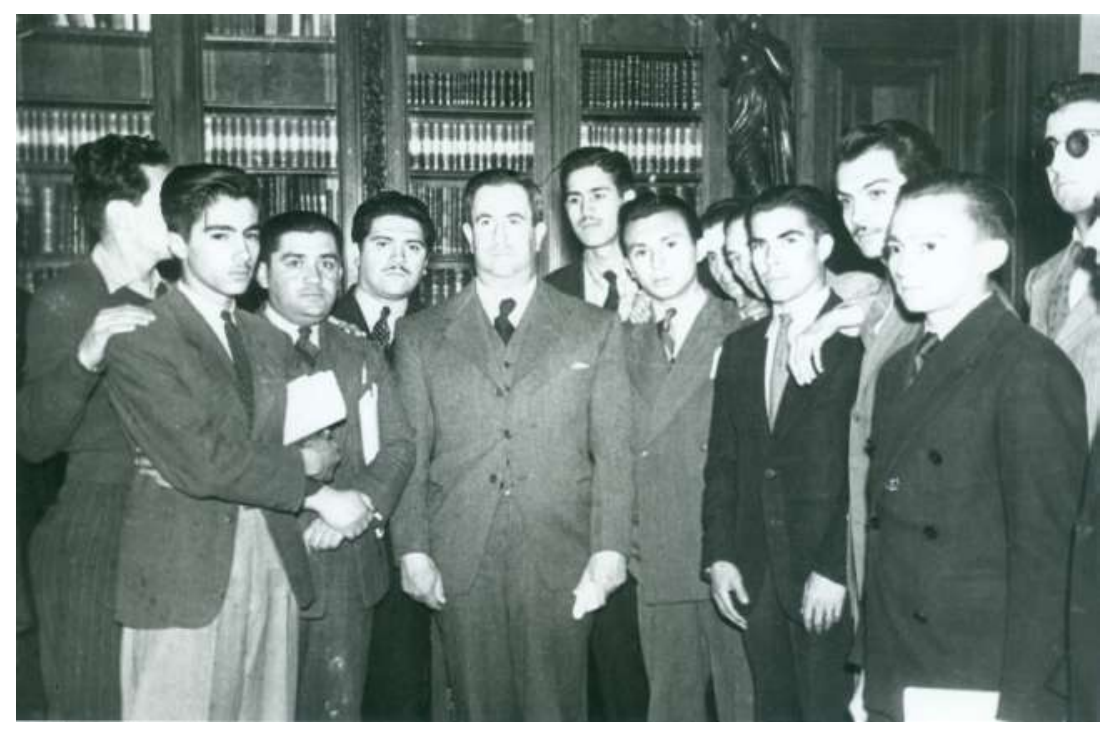

Fotografía 2. El presidente Manuel Ávila Camacho recibe a una comisión de alumnos, Palacio Nacional. AHC-IPN, Fototeca, 10 de marzo de 1942.

\section{[...]. ¿Qué finalidades atribuye la Secretaría de Educación Pública al Instituto Politéc- nico Nacional como unidad educativa de carácter superior? ${ }^{14}$}

El secretario contestó que en el IPN "se gradúan arquitectos, pero no se forman maestros de obras ni se capacitan obreros calificados" y puntualizó que en la enseñanza técnica se debían preparar cuadros de trabajadores empíricos, obreros calificados, maestros de taller para fungir en las distintas áreas productivas, y técnicos profesionales; y señaló lo que él consideraba un error mayúsculo: "hay también enseñanza universitaria en el Politécnico, porque con él se quiso hacer una especie de competencia a las Universidades". Véjar Vázquez expresó que la educación técnica-tecnológica debía circunscribirse a "preparar a los elementos humanos para que intensifiquen la producción económica". ${ }^{15}$ En su réplica se aprecia nítidamente la intención del gobierno de separar las escuelas técnicas, eliminando su carácter nacional.

Aunque en el informe de labores de la SEP de 1943 se reconocía la importancia de la enseñanza técnica, la cual "se divulgó por medio de escuelas vocacionales; preparatorias fronterizas normales; Superior de Ingeniería y Arquitectura; Federal de Industrias Textiles;

${ }^{14}$ Diario de los debates de la Cámara de Diputados del Congreso de los Estados Unidos Mexicanos, 30 de diciembre de 1942.

15 Idem. 
Superior de Ingeniería Textil; Nacional de Ciencias Biológicas; Nacional de Medicina Homeopática; Superior de Ciencias Económicas, Administrativas y Sociales" (SEP, 1976: 251), el Politécnico siguió sin ser mencionado por los funcionarios de la educación.

Lo interesante aquí es que, tras la agresión del Estado en contra de los estudiantes en marzo de 1942, sobrevino un alud de protestas dirigidas al presidente Ávila Camacho por parte de sindicatos, grupos estudiantiles, maestros, campesinos y ciudadanos en general de diversas partes de la República quienes denostaron el hecho de la represión. Los planteles Azcapotzalco y Santo Tomás de la Escuela Normal se declararon en huelga el 10 de marzo en solidaridad con los estudiantes del Politécnico, y el Bloque de Federaciones Estudiantiles de Escuelas Técnicas, Normales para Hijos de Trabajadores y Campesinos convocó a la sociedad a una marcha que partiría del monumento a la Revolución el jueves 12 de marzo.16

Este es también el sentido de muchas de las cartas y telegramas que ciudadanos de diversas partes del país le enviaron al presidente Ávila Camacho y a los diarios de la Ciudad de México tras los hechos violentos en el Zócalo. A continuación presentamos tres ejemplos. La Sociedad de Vecinos de Tixtla, Guerrero expresaba:

Nosotros que tenemos estudiando a nuestros hijos en las diferentes escuelas de esa capital, a costa de innumerables sacrificios, porque somos pobres y humildes, nos sentimos sumamente lesionados en nuestras aspiraciones, al conocer que una orden suya sobre el particular, viene a redundar en perjuicio grave de miles y miles de muchachos que han soñado en un futuro mejor y que al llevarse a cabo tal determinación, quedarían suspendidos en sus estudios quedando a sí mismo desamparados de los beneficios de ese Supremo Gobierno que la propia Revolución le ha impuesto impartir a la clase necesitada. ${ }^{17}$

El texto refleja el sentir popular en el interior de la República respecto a que el Politécnico ofrecía capacitación rápida a través de cursos técnicos y formación profesional los cuales representaban oportunidades de mejoría económica y cultural, por medio de la educación, a los hijos de familias de sectores desfavorecidos. El siguiente es un escrito de la Sociedad Cooperativa de Reboceros de la Piedad, Michoacán:

Creemos que los muchachos que estudian en las aulas de un Instituto formado por la Revolución, al llevar su movimiento de huelga y de la manifestación del día 6, fue con pleno derecho que les conceden las leyes mexicanas, y sólo a un ofuscado como Véjar Vázquez, elemento que ha dado muestras de ser un cafre, al ordenar se masacraran a los estudiantes [...]. Por ello venimos a pedir a usted, tome las medidas del

16 "Continúa sin solución la huelga en las escuelas", El Universal, 10 de marzo de 1942, p. 1.

${ }^{17}$ AHC-IPN (1941-1942), Compendio de noticias con referencia al movimiento estudiantil de 1942, t. III Ciudad de México, Hemeroteca, p. 205. 
caso y resuelva satisfactoriamente en bien de los estudiantes pobres que son víctimas de la reacción. ${ }^{18}$

Por su parte la Comisión Organizadora del Partido Comunista de México, sección Guerrero manifestaba: "Intentar aplastar a la fuerza un acto noble y justo de los estudiantes del Politécnico, que como base tiene una mal encauzada política educativa por el titular del Ramo, significa despertar el odio de las masas más oprimidas, pero más fuertes del pueblo, en contra del Lic. Octavio Véjar Vázquez, quien, por su actuación como representante de la Secretaría de Educación, dio origen a esta serie de anormalidades". ${ }^{9}$

El Politécnico tenía presencia en el interior del país gracias a que, al momento de su puesta en marcha, estaban en funcionamiento las escuelas prevocacionales en las ciudades de Campeche, Campeche; Culiacán, Sinaloa; Durango, Durango; Guadalajara, Jalisco; Hermosillo, Sonora; Jiquilpan, Michoacán; Juchitán, Oaxaca; Las Casas, Chiapas; Puebla y Teziutlán, Puebla; Tuxtla Gutiérrez, Chiapas; además de la Ciudad de México. Respecto a éstas las autoridades educativas señalaron: "[se planeó] que sus puertas de entrada estuvie-

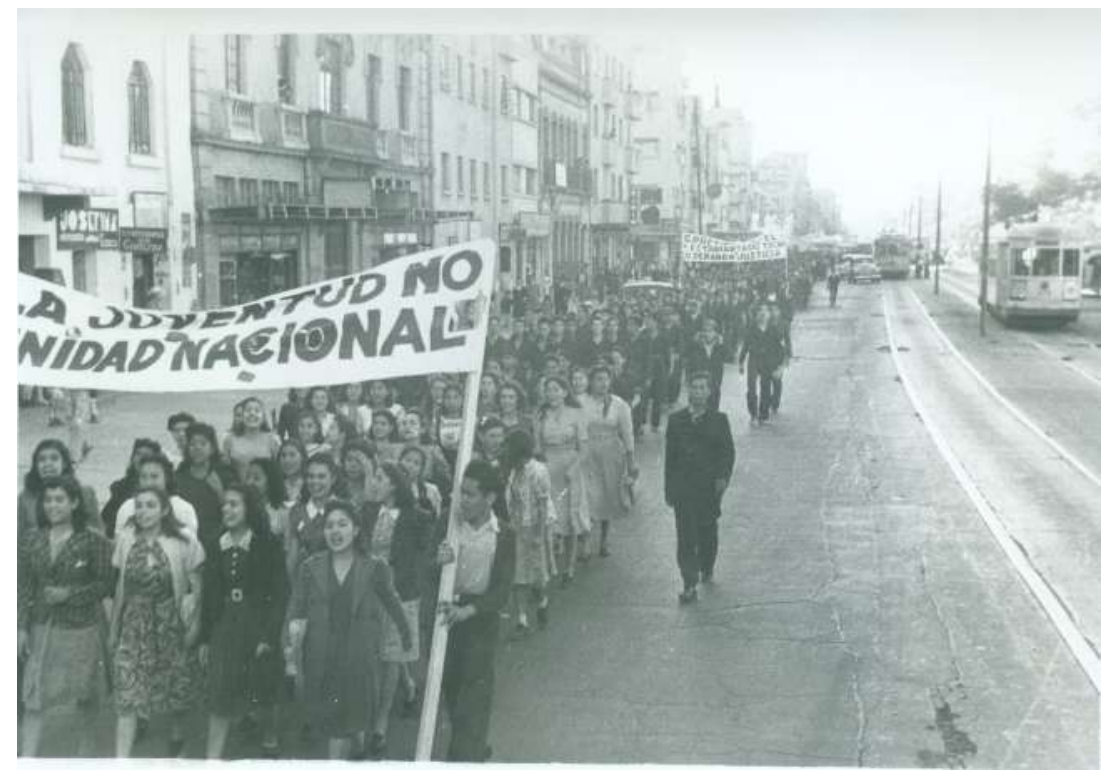

Fotografía 3. Manifestaciones de mujeres estudiantes en apoyo del movimiento politécnico. AHC-IPN, Fototeca, 12 de marzo de 1942.

\footnotetext{
${ }^{18}$ AHC-IPN (1941-1942) Compendio de noticias con referencia al movimiento estudiantil de 1942, t II, Ciudad de México, Hemeroteca, p. 79.

${ }^{19}$ /dem, p. 91.
} 
ran abiertas para todos los aspirantes a hacer una carrera técnica en cualesquiera de las regiones del país. [...] El alumnado que ingrese a las escuelas de este tipo, puede proseguir, mediante su paso por Escuelas Vocacionales, hacia todos los estudios profesionales del Instituto Politécnico Nacional" (SEP, 1937: 83).

Estos ejemplos forman parte del discurso histórico-político construido por el gobierno cardenista, asumido y reelaborado por parte de los sectores populares, agrupados por el propio Estado en corporaciones políticas. Para la comunicación con el gobierno de Ávila Camacho estos grupos emplearon el único canal que el sistema corporativo les permitía, la voz de la agrupación, fuera ésta sindical, campesina, estudiantil o de profesionistas.

Las asociaciones como la Confederación de Jóvenes Mexicanos, la Federación de Profesionistas Veracruzanos, Juventudes de México y América, el Sindicato de Trabajadores de la Secretaría del Trabajo, el Sindicato Unión Nacional de Trabajadores de la Enseñanza (SUNTE), el Sindicato de Trabajadores de la Enseñanza de la República Mexicana (STERM), ${ }^{20}$ y algunas más, se pronunciaron pacíficamente en favor de los estudiantes y fueron escuchadas, entre otras razones, por el hecho de que el gobierno necesitaba mantener ante ellas su investidura de heredero de la Revolución, so pena de perder legitimidad.

El 20 de abril de 1942 el presidente de la República nombró a José Laguardia Núñez jefe del Departamento de Enseñanza Técnica —-debido a que había desaparecido de la estructura el puesto de director general del IPN, aunque en la práctica, Laguardia asumió esa función-. A principios de 1943 Laguardia fue ratificado en el cargo de "jefe del Instituto Politécnico". En mayo se resolvió el conflicto de la ENCB al equipararse la carrera de medicina rural con la carrera de médico militar, mediante el acuerdo presidencial 294, lo que motivó una ceremonia de agradecimiento organizada el 4 de agosto en Santo Tomás, a la cual asistieron el presidente de la República, el secretario de Educación e incluso el expresidente Lázaro Cárdenas (Calvillo y Ramírez (2006: 271-276). En diciembre de ese año Octavio Véjar Vázquez dejó la dirección de la SEP a Jaime Torres Bodet (1943-1946), y fue el nuevo secretario quien planteó que la escuela debía ser una para todos los mexicanos. Con la intención de fomentar la unidad nacional consideraba que la educación debía desprenderse de todo rastro de adoctrinamiento político y apagar cualquier idea de lucha de clases, construyendo un ciudadano proclive al trabajo.

En enero de 1944 el gobierno renunció definitivamente a la pretensión de suprimir el instituto. El presidente Ávila Camacho nombró al reconocido científico Manuel Sandoval Vallarta nuevo director del IPN y promulgó el Reglamento Provisional del Instituto Politécnico Nacional en el que se apuntaba: "Una educación técnica coordinada y una eficiente investigación industrial constituyen necesidades vitales para un país como el nuestro [...]. Por la

20 "Continúa sin solución la huelga en las escuelas", El Universal, 10 de marzo de 1942, p. 11. 
naturaleza de sus labores y por las sumas consagradas a su creación y a su mantenimiento, el Instituto Politécnico Nacional es, sin duda, el más importante de los planteles con que contamos para lograr el propósito antes mencionado". ${ }^{21}$ Así, la institución obtuvo el anhelado reconocimiento oficial y como muestra de que el gobierno incluía nuevamente al Politécnico en el proyecto educativo, también le fueron restituidas las escuelas prevocacionales de la capital.

El $1^{\circ}$ de octubre de 1945 se emitió el Reglamento de la Ley Reglamentaria de los artículos $4^{\circ}$ y $5^{\circ}$ constitucionales, ley creada por la Dirección General de Profesiones, dependencia de la SEP, a cargo de emitir títulos profesionales. El IPN quedó inscrito en esta ley, con lo cual se subsanó la omisión del mismo en la Ley Orgánica de Educación Pública de 1942.

\section{Importancia estratégica del Politécnico en los planos ideológico y material}

Elementos del discurso político-educativo que inició en plena lucha revolucionaria —en la década de 1910 - , fueron conservados por el gobierno de Manuel Ávila Camacho. Permanecieron inalterables los principios educativos: gratuidad y obligatoriedad de la enseñanza básica, y se mantuvo a las asociaciones religiosas fuera de las escuelas públicas. Junto con esa parte dogmática se conservaron los contenidos de los planes y programas sustentados en los avances de la ciencia y la tecnología. Estas características de la educación revolucionaria se habían convertido en elementos necesarios e intocables para mantener en calma a los grupos populares, a lo cual debemos sumar la enseñanza técnica como opción educativa para los hijos de trabajadores.

Tras el movimiento estudiantil de 1942 las autoridades políticas y educativas avilacamachistas reconocieron al IPN como constructor político-educativo, asimilado por los grandes sectores populares como uno de los pocos frutos recibidos de la Revolución hasta ese momento. Entre algunas de las instituciones más representativas del cardenismo que fortalecían la legitimidad del Estado (la CTM, la CNC, el ejido, Pemex, la Comisión Federal de Electricidad, los Ferrocarriles Nacionales de México, el Instituto Nacional de Antropología e Historia, el Instituto Indigenista Interamericano, etcétera) se encontraba el Instituto Politécnico Nacional al cual Cárdenas había presentado como una institución educativa para el pueblo, surgida de la Revolución mexicana y proyectada desde la redacción misma de la Constitución de 1917.

Otro elemento que perduró con la reorientación de los fines del Estado, a inicios de la década de 1942, fue la reproducción del "discurso revolucionario", que sirvió para fortalecer el

21 "Reglamento Provisional del Instituto Politécnico Nacional de 1944", DOF, 17 de febrero de 1944, p. 2. 
pacto social que logró el gobierno de Lázaro Cárdenas con obreros y campesinos. Se continuó hablando de acciones impulsadas por el gobierno en favor de las clases necesitadas y al hacerlo, resultó fortalecida la relación paternalista del Estado con los sectores marginados.

A partir del gobierno de Ávila Camacho, la organización corporativa de los sectores sociales se constituirá en mecanismo para desarmar la movilización de los sectores productivos, ahora subordinados al proyecto del Estado de fortalecer la economía capitalista "nacional", coordinados por un sistema político presidencialista. Obreros y campesinos participaban en el nuevo modelo como generadores de riqueza que se orientaba a la industrialización y a la acumulación de capital, en beneficio de la pequeña y gran burguesía.

Por otra parte, además del control ideológico, el instituto era indispensable para el Estado en la implementación del proyecto de modernización material del país que ya estaba en marcha, y en el que la educación técnica era vital, considerando que "los problemas que planteará a México el periodo de articulación económica al término de la guerra exigirán de nosotros una capacidad constructiva cuya sola previsión nos impone el deber de conformar en nuestras escuelas los equipos técnicos destinados al rendimiento futuro de la República" (SEP, 1946: 31). Así lo reconoció el presidente Ávila Camacho en su informe del $1^{\circ}$ de septiembre de 1945: "Se impone a nosotros un plan nacional para conseguir, en lo concerniente a la educación, más escuelas y más maestros, y en lo que atañe a la economía, un rendimiento agrícola más fructuoso y una industrialización provechosa de los recursos naturales que no explotamos" (SEP, 1976: 259-260).

La necesidad de educación expresada por el presidente implicaba la valoración del IPN como institución educativa de carácter técnico-tecnológico y esa consideración especial se reflejó en el presupuesto asignado, que fue en aumento a partir de 1943, incluso superó al de la universidad, en 1948, cuando al Politécnico se le asignaron 15700000 pesos, de los cuales dos millones fueron invertidos en la construcción de la Ciudad Politécnica en Santo Tomás, en la Ciudad de México.

\section{Conclusiones}

Con el cambio de administración y de política educativa, en 1940, algunos funcionarios compartieron con sectores medios y altos de la sociedad mexicana el recelo hacia el Instituto Politécnico Nacional, considerando que para dispersar enconos era necesaria su desaparición y asignar a sus escuelas la función de formadoras de cuadros técnicos, disminuyendo la educación profesional que impartían y encomendando esa tarea a la universidad. Como parte del sistema educativo nacional a cargo del Estado, se suponía que todos sus componentes debían reproducir la organización político-social y económica impulsada por la nueva política del 
gobierno. Fue en ese momento que el sentido social que arropó la construcción del IPN quedó desfasado ante el interés de la élite y del Estado por fortalecer el individualismo y el capitalismo.

Sin embargo, debido a que el IPN se había convertido en una pieza representativa de la justicia social lograda por la Revolución mexicana, - gracias al discurso y las acciones del gobierno cardenista, a la presión de las organizaciones y al hecho de que el instituto representó una poderosa herramienta para construir la modernidad nacional y para la industrialización de los sectores productivos, fue que las autoridades recapacitaron respecto a su aniquilación.

Después ocurrieron adecuaciones. De acuerdo con la política de Jaime Torres Bodet, y para minimizar fricciones sociales y entre la comunidad estudiantil, las autoridades del Politécnico modificaron su discurso el cual colocaba a las escuelas del IPN como centradas en atender a los jóvenes pertenecientes a los bajos niveles de la sociedad. Ese es el sentido del siguiente texto oficial:

\footnotetext{
Por su origen familiar, los politécnicos son como los alumnos de otra escuela cualquiera, de esas a las que acude el mexicano "estándar". No es el reducido grupo de hijos de gente rica, que buscan el privilegio de una educación rodeada de todas las facilidades. Pero tampoco el conglomerado de los desheredados que buscan exclusivamente el arrimo en una institución que los eduque de caridad. Ningún extremo en ningún sentido. Domina en número el joven de clase media (Centro Recreativo Estudiantes Politécnicos, 1952: 9 y 10).
}

En lugar de ello, en adelante el gobierno trataría de quitarle al IPN el sello de educación exclusiva para trabajadores y sectores pobres. En las siguientes décadas el crecimiento económico del país abriría numerosos espacios para los egresados de todas las escuelas públicas; de tal modo que obtener un título en el IPN se convirtió en la posibilidad real de ascenso social, e incluso de ingresar al círculo de las clases privilegiadas.

\section{Fuentes}

\section{Archivos consultados}

AHC-IPN Archivo Histórico Central del Instituto Politécnico Nacional (1941-1942), "Compendio de noticias con referencia al movimiento estudiantil de 1942", 3 t., Archivo Histórico Central del Instituto Politécnico Nacional, Ciudad de México, Hemeroteca.

AHC-IPN (1942), "Acuerdo de Roberto T. Bonilla, subsecretario de Educación Pública, al director general de administración, 14 de enero", Ciudad de México, Fondo Colección Especial, Sección Wilfrido Massieu.

Archivo Histórico de la Escuela Superior de Ingeniería Mecánica y Eléctrica (1952), "Folleto Centro Recreativo Estudiantes Politécnicos", Ciudad de México, Fondo Tomás Guzmán Cantú, caja 6, exp. $s / n$ 
AH-ENCB Archivo Histórico Escuela Nacional de Ciencias Biológica (1938), "2 Congreso de Higiene Rural", Ciudad de México, Área Documental, caja 1, exp. Leopoldo Ancona Hernández.

Fuentes hemerográficas

DOF (Diario Oficial de la Federación) (1934), "Decreto que reforma el Artículo $3^{\circ}$ y la fracción xxv del 73 constitucionales", Diario Oficial de la Federación, 13 de diciembre de 1934.

(1942), "Ley Orgánica de Educación Pública, reglamentaria de los artículos 30; fracción I; 73, fracciones x y xxv; y 123, fracción xII, de la Constitución Política de los Estados Unidos Mexicanos", Diario Oficial de la Federación, 23 de enero de 1942.

(1944), "Reglamento Provisional del Instituto Politécnico Nacional de 1944", Diario Oficial de la Federación, Ciudad de México, 17 de febrero de 1944, pp. 2-5.

Diario de los Debates de la Cámara de Diputados del Congreso de los Estados Unidos Mexicanos (1942), Ciudad de México, 30 de diciembre de 1942.

El Nacional (1937), "Los cursos en las Escuelas Politécnicas se inauguraron ayer en todos los planteles que dependen del Instituto Politécnico Nacional", El Nacional, 21 de febrero de 1937.

(1942), "El ejecutivo dará una solución justa a las peticiones escolares", El Nacional, miércoles 11 de marzo de 1942, p. 1.

El Universal (1942a), "Continúa sin solución la huelga en las escuelas", El Universal, 10 de marzo de 1942, p. 1.

(1942b), "Los causantes de la agitación estudiantil", editorial, El Universal, 6 de marzo de 1942. (1942c) "Muchos alumnos de las escuelas técnicas están contra la huelga", El Universal, 6 de marzo de 1942, p. 4.

(1942d),"Zafarrancho entre policías y estudiantes; el saldo fue de 2 muertos y 18 heridos", El Universal, 7 de marzo de 1942, p. 1 .

(1942e),"Cuando luche la juventud, que sea por su decoro", El Universal, 8 de marzo de 1942, p. 1.

Bibliografía

Calvillo, Máx y M. L. Ramírez (2006), Setenta años de historia del Instituto Politécnico Nacional, t. I, Dirección de Publicaciones/Instituto Politécnico Nacional, México.

Civera, Alicia (2013), La escuela como opción de vida. La formación de maestros normalistas rurales en México. 1921-1945, El Colegio Mexiquense, A.C.-Fondo Editorial Estado de México/Gobierno del Estado de México, México.

Departamento de Enseñanza Técnica, Industrial y Comercial (1933), Reglamento de alumnos para las Escuelas Preparatorias Técnicas y de Estudios Técnicos Superiores, que integran la Escuela Politécnica Nacional, Secretaría de Educación Pública/Departamento de Enseñanza Técnica, Industrial y Comercial, México.

Gómez Nashiki, Antonio (2003), "El movimiento estudiantil mexicano. Notas históricas de las organizaciones políticas, 1910-1971", Revista Mexicana de Investigación Educativa, vol. 8, núm. 17, enero-abril, pp. 187-220

González, Luis (2005), Historia de la Revolución Mexicana, 1934-1940: los días del presidente Cárdenas, Centro de Estudios Históricos/El Colegio de México, México.

Guevara, Gilberto (1980), "La educación superior en el ciclo desarrollista de México", Cuadernos Políticos, núm. 25, julio-septiembre, pp. 54-70.

(1984), "El IPN y la reforma cardenista", en Oscar Moharb B. (comp.), Crisis y contradicciones en la educación técnica de México, Gaceta, México, pp. 53-72. 
Junco, Alfonso (1942), "En busca de un renacimiento. Habla el ministro de Educación", El Universal, 6 de marzo de 1942, p. 5.

Knight, Alan, (1988), "México, 1930-1946", en Leslie Bethell (ed.), Historia de América Latina, vol. 13, Cambridge University Press-Crítica, Barcelona, pp. 52-83.

López, Fernando (2011), "Los inicios de la ENAH: 1937-1942", en Alejandro Villalobos (coord.), Escuela Nacional de Antropología e Historia, Instituto Nacional de Antropología e Historia-Consejo Nacional para la Cultura y las Artes, México, pp. 21-41.

Monteón, Humberto (1986), "El Instituto Politécnico Nacional: proyecto educativo revolucionario del cardenismo", Revista de la Educación Superior, vol. 15, núm. 58, abril-junio, pp. 1-12.

Ordorika, Imanol (2006), La disputa por el campus. Poder, politica y autonomía en la UNAM, Centro de Estudios sobre la Universidad/Universidad Nacional Autónoma de México-Plaza y Valdés, México.

Pérez, Adolfo (1984), 50 años de investigación en la Escuela Nacional de Ciencias Biológicas, 1934-1984, una valoración crítica a la luz de su evolución histórica, Escuela Nacional de Ciencias Biológicas/Instituto Politécnico Nacional, México.

Rodríguez, Ma. de los Ángeles y Max Krongold (1988), 50 años en la historia de la educación tecnológica, Instituto Politécnico Nacional, México.

Ruiz, Estela (2004), Ingenieros en la industria manufacturera, Centro de Estudios sobre la Universidad/ Universidad Nacional Autónoma de México-Plaza y Valdés, México.

Secretaría de Educación Pública(SEP) (1932), Memoria relativa al estado que guarda el Ramo de Educación Pública el 31 de agosto de 1932, t. I, Secretaría de Educación Pública-Talleres Gráficos de la Nación, México.

(1934), Memoria relativa al estado que guarda el Ramo de Educación Pública, 31 de agosto de 1934, t. ı, Secretaría de Educación Pública, México.

(1937), Memoria de la Secretaría de Educación Pública. De septiembre de 1936 a agosto de 1937, Presentada al H. Congreso de la Unión por el Licenciado Gonzalo Vázquez Vela Secretario del Ramo, t. I, Secretaría de Educación Pública, D.A.P.P., México.

(1939), Historia gráfica del IPN, vol. I, Anuario 1939, Secretaría de Educación Pública/Departamento de Enseñanza Técnica-Departamento de Educación Audiovisual/Instituto Politécnico Nacional, México.

(1941), La educación Pública en México, desde el $1^{0}$ de diciembre de 1934 hasta el 30 de noviembre de 1940, t. I y II, Secretaría de Educación Pública, México.

(1944), Memoria de la Secretaría de Educación Pública. Labores desarrolladas en el periodo comprendido entre el día I de septiembre de 1943 al 31 de agosto de 1944, vol. II, Secretaría de Educación Pública, México.

(1946), La obra educativa en el sexenio 1940-1946, Secretaría de Educación Pública-Talleres Gráficos de la Nación, México.

(1976), México a través de los mensajes presidenciales, la educación pública, t. II, Secretaría de Educación Pública-Secretaría de la Presidencia, México.

Urquidi, Víctor (2005), Otro siglo perdido. Las políticas de desarrollo en América Latina (1930-2005), Fondo de Cultura Económica-El Colegio de México, México.

Andrés Ortiz Morales es profesor de educación superior adscrito al Archivo Histórico del Instituto Politécnico Nacional. Doctor en Historia y Etnohistoria por la Escuela Nacional de Antropología e Historia (2006-2011), realizó estudios de maestría en la misma institución (2004-2006) y de licenciatura en el área de Ciencias Sociales en la Escuela Normal Superior 
de México (1996-2000). Su línea de investigación es la educación pública como ámbito de desarrollo de la investigación científico-tecnológica en México. Sus más recientes publicaciones son: De la ciencia aplicada a la investigación científico-tecnológica. ESIME (1935-1961), Instituto Politécnico Nacional, Dirección de Publicaciones, México, 2016; y "Capacitación en el sistema educativo, crisis y revaloración (1982-1994)", UPIICSA. Investigación interdisciplinaria, Instituto Politécnico Nacional, $3^{\text {a }}$ ed., vol. 2, núm. 1, enero-junio de 2016.

Recibido: 28 de marzo de 2017. Aceptado: 13 de agosto de 2017. 